\title{
Mechanical properties of polyester composite reinforced fibers hybrid bamboo - Musa acuminata stem fiber as raw material of rear bumper vehicle
}

\author{
Sujita Darmo Darmo * and Rudy Sutanto Sutanto \\ Department of Mechanical Engineering, Faculty Engineering, University of Mataram, Majapahit Street 62, Mataram \\ 83125, Indonesia.
}

Global Journal of Engineering and Technology Advances, 2021, 08(03), 001-007

Publication history: Received on 28 July 2021; revised on 30 August 2021; accepted or 1 Septe her 2021

Article DOI: https://doi.org/10.30574/gjeta.2021.8.3.0123

\begin{abstract}
In the present study fiber hybrid (bamboo fiber and Musa acumin ste. fibers (MASF) were reinforced polyester composite, for enhancing mechanical properties. The natural fib s composit o gained its importance due to its low cost, substitute for artificial fiber, low price, able to reduce soun environ entally friendly, has a low density, good fit and ability to absorb impact energy. It's possible to use it as a study aims to investigate the tensile stress-strain, elas - 1 ty ndur a the microstructure of fracture of polyester fiberglass composite reinforced bamboo fiber and MA hy ia olyester matrix type 157 is used BQTN and G3253T, MEKPO catalyst. The mechanical properties test y carri d out oy universal testing machine (UTM) test instrument, observing the microstructure of the occurrene f fract $a b$ using scanning electron microscope (SEM). The test specimens produced the vacuum infusion hea results of this study indicate that the addition of more MASF and the outer layer content can increase the te stre the elasticity modulus of polyester composites reinforced MASF with woven, random arrangement is better th that o polyester composites reinforced bamboo fiber. So that in the manufacture of polyester composit cild fiber the use of more MASF than bamboo fiber can provide better tensile strength and elasticit mod us The configuration with the outer MASF layer can also increase the value of tensile strength, elast odu the ecimen compared to the configuration with bamboo fiber outside. The microstructure of fra are for ber th configuration of MASF content there are long fibers and also in bamboo fibers there are fib $s$ that ar pulled out. The diameter of the bamboo fiber reinforcement is larger so that the load transfer is not as eft ive aninum
\end{abstract}

Keywords: Polyester co vosites; Bamboo fiber; Musa acuminata stem fiber; Hybrid; Tensile stress-strain; Rear bumper

\section{Introduction}

Recently, the automotive industry tends to use composite materials for its exteriors such as bumpers, extenders and followers. The reasons are composite materials, low density, light weight and corrosion resistance. The polyester matrices composite reinforced natural fibers present a great potential application in the automobile industry, especially in the bumper of automobiles, bus and trucks.

The future perspective for the use of natural fibers is very good also in other areas. For instance, the textile industry is now with expansion international market. In the last years, use of natural fibers as, coconut, sisal, rami, sugar-cane pulp, jute and pineapple as reinforcement in polymeric material had an accelerated growth. They are source of renewable natural resource [1].

\footnotetext{
${ }^{*}$ Corresponding author: Sujita Darmo Darmo

Department of Mechanical Engineering, Faculty Engineering, University OF Mataram, Majapahit Street 62, Mataram 83125, Indonesia.
}

Copyright (C) 2021 Author(s) retain the copyright of this article. This article is published under the terms of the Creative Commons Attribution Liscense 4.0. 
Natural fibers present low cost, are biodegradable, recycled, no poisonous and can be incinerated. They are being used as reinforcement in polymeric and substitute synthetic fibers partially as asbestos, Kevlar, boron, carbon, nylon and glass. In spite of these good mechanical characteristics, they present a high cost, are abrasive to the processing equipment's, possess high density, generate products with very high recycling cost, besides some of those fibers commit human health $[2,3]$.

Matrices have as main purpose to transfer the stresses imposed on the composite material to the fibers, as well to serve as a support and protection of the fibers. Generally, composite matrices of thermosetting resins are used because they have great mechanical properties and dimensional stability. They are also resistant to chemical attack and have high thermal resistance. According to $[4,5]$ the matrix has three main functions which are: to protect the surface of fibers from damage by abrasion that would lead to fracture, adhere in the surface in order to transfer the force has applied to the same fiber, to separate the fibers each other in order to improve the resistance of propagation of transverse cracks to other fibers.

The rear bumper is one part of the vehicle that has a very important role, apart from being aerodynamic and aesthetically pleasing to attract consumers, the bumper also functions as a collision da, er from the rear that occurs in the vehicle. Therefore, the material used as the rear bumper often gets damaged durin. collision, so it requires a material that has good impact toughness, is light, ductile and corrosion resistant. It is cima that the annual world car production rate will reach 76 million vehicles per year by 2020. New regulations si $\mathrm{h}$ as the End of Life Vehicles (ELV) regulations are forcing car manufacturers to consider the environmental impact their p fauction and possibly shift from the use of synthetic materials to the use of agro-based materials. or properties and certain manufacturing limitations currently limit the use of agro-based material to n-cructural and semi-structural automotive components. The hybridization of natural fiber with ${ }^{2} s$ fi $r$ provic $s$ a method to improve the mechanical properties over natural fibers alone $[6,7]$. The hybridiza of method to improve the mechanical properties over natural fibers alone, discussed in detail in [7]. This research is focused on a hybrid of kenaf/glass fiber to enhance the desir a mechan roperties for car bumper beams as automotive structural components with modified sheet molding ompour (SMC). A specimen without any modifier is tested and compared with a typical bumper beam material calle rlass $m$ thermoplastic (GMT). The results indicate that some mechanical properties such as tensile strengt ${ }^{\prime}$ ' $g^{\prime}$ s slexural strength and flexural modulus are similar to GMT, but impact strength is still low, and sh ws th tential for utilization of hybrid natural fiber in some car structural components such as bumper beams

Referring to the study [8, 9], the hybrid g s / car n compunce bumper beam was designed and manufactured via the design optimization process combined witt impa analysis. The glass/carbon mat thermoplastic (GCMT) composite was devised to substitute for the conventiona 'ass ma hermoplastic (GMT) for reducing the weight of bumper beam. For the design optimization, the mect rop of GCMT were predicted and the optimal design of bumper beam was performed with the impact sim latio Based on the final design, the real bumper beam was manufactured and its impact performances we easu d a was ound that the optimally designed GCMT bumper beam had $33 \%$ less weight compared to th conve iona. MT b hper beam while having the improved impact performances.

The works [10] des opt bumper systems has focused on one single impact loading. This study aims to address this issue by oping a multi objective optimization approach, in which multiple impact loading scenarios are considered to optim. the intrusion and energy absorption of the bumper system. Modeling of real-life impact scenarios is first validated comparing the numerical results with the experimental data. The optimization results indicate that the Pareto solutions largely depend on the assignment of weight factors to different load cases. In order to generate more reasonable results, the National Automotive Sampling System-Crashworthiness Data System (NASSCDS) database is also used to determine the weighting factors for each loading case. The study help gain insights into the design optimization of a bumper system for real-life automotive engineering.

Thus, it should be noted that there is no data found in the literature that would explain the possibility of using hybrid fibers (bambo fiber and MASF) as a polyester composite reinforcement, so that its mechanical properties are better, suitable for use as a bumper material. This study aims to determine the mechanical properties (tensile stress-strain, elasticity modulus) of polyester composite reinforced hybrid fibers (bambo fiber and MASF), for the feasibility of these composites as an alternative solution to the replacement material for Multi-Purpose Vehicle (MPV) bumpers. 


\section{Material and methods}

\subsection{Materials}

The research materials are Bamboo fiber, Musa acuminata stem fiber, Polyester matrix type 157 BQTN and G3253T, MEKPO catalyst, $5 \% \mathrm{KOH}, 5 \% \mathrm{NaOH}$, shawn in Figure 1 . The equipment used are Universal Testing Machine (Tensilon RTG-1310), Scanning Electron Microscope (SEM FEI inspect S50), vacum pump, composite mold made of silicon, with a thickness of $6 \mathrm{~mm}$ standard and composite fabrication equipment.

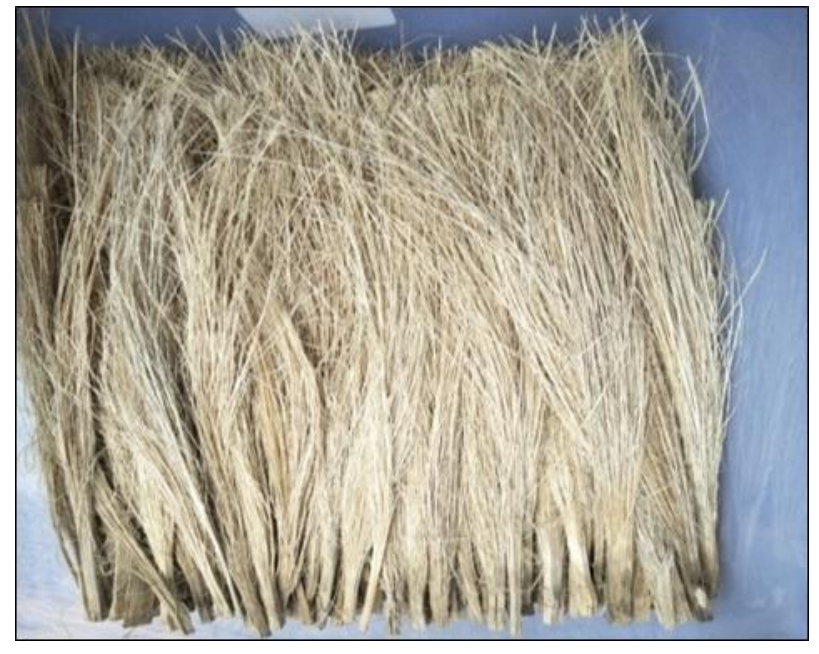

A

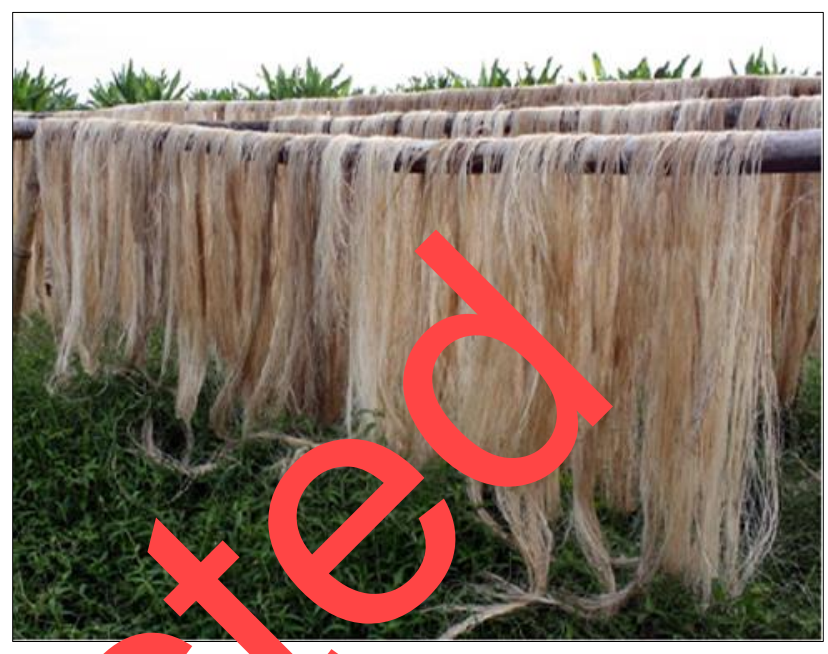

$\mathrm{B}$

Figure 1 a. Bamboo Fiber. b. Musa ac ninatas m fiber (MASF)

\subsection{Methods}

The method is done by the fiber's hybrid (Bambo fib, , ASF) , ated alkaline with soaking with $8 \%$ NaOH for 120 minutes. Composite fabrication using the vacuum ifusion hold $g$ method. The specimen are made arranging bamboo fiber and MASF (woven, random), with op figh tions B Specimens and tensile testing procedures fer to ASTM D3039 standard [4] and the microstructure of material fracture observation used Scanning Electron 1 rosco ${ }_{\mathrm{P}}(\mathrm{SEM})$.

\section{Results and discussion}

\subsection{The mechanic proper' ss of poryoucer matrix composite reinforced fiber hybrid}

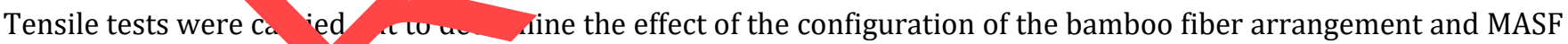
on the tensile propert of the hybrid fiber reinforced polyester composite. From the results of the tensile test, the values and average valu of tensile strength, tensile strain, and modulus of elasticity are obtained in various configurations of BBBBBB, B . $3 M B M$, MBMBMB, BBMMBB, and MMMMMM, with random and woven arrangements, as shown in Figures 2 to 4 .

From the results of the tensile test, the tensile strength of the specimens was obtained by, arrangements variations of random and woven in the various configurations of BBBBBB, BMBMBM, MBMBMB, BBMMBB, and MMMMMM as shown in Figure 2. Tensile strength with random fiber arrangement, higher than with woven fiber. Highest tensile strength by $175,85 \mathrm{MPa}$ on configuration MMMMMM (MASF fiber). And the lowest is 25,21 MPa with woven fiber arrangement on the configuration BBBBBB. The tensile strength optimum of polyester composite reinforce fiber hybrid by $72,48 \mathrm{Mpa}$ with woven fiber arrangement on configuration MMBBMM.

The tensile strain at fracture for hybrid composites with random MASF was higher than woven arrangement, as shown in Figure 3. However, the effect of MASF or bamboo fiber location, by turns configurations (MBMBMB, BMBMBM) or block configuration (BBMMBB, MMBBMM) was not showing a significant difference. The highest tensile strain by 0,0235 random arrangement, configuration MMBBMM and the lowest by 0,0093 woven arrangement, configuration BMBMBM. 


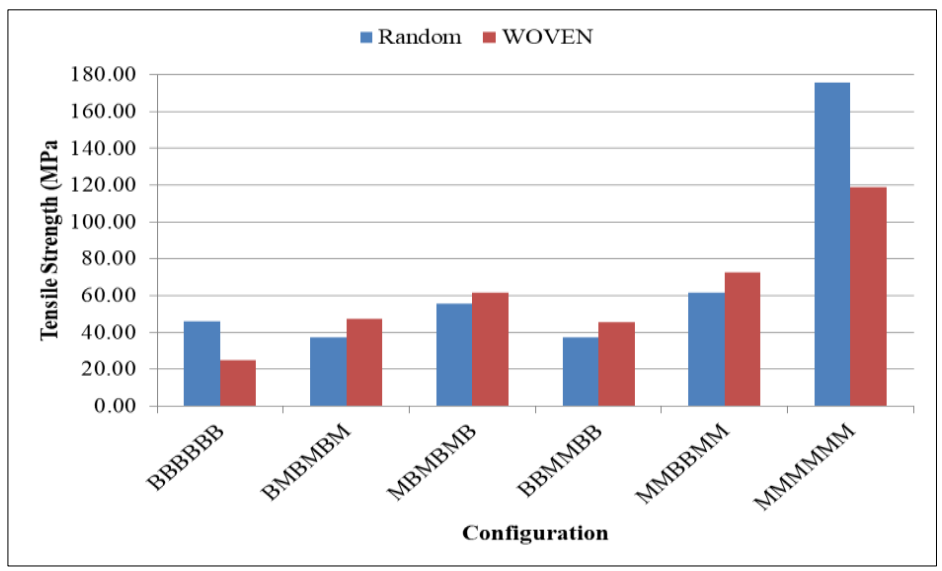

Figure 2 Tensile Strength of polyester composite reinforced fiber hybrid (ba boo fiber-MASF)

gure 3 Tensile Strair of pol ster connposite reinforced fiber hybrid. (Bamboo fiber-MASF)

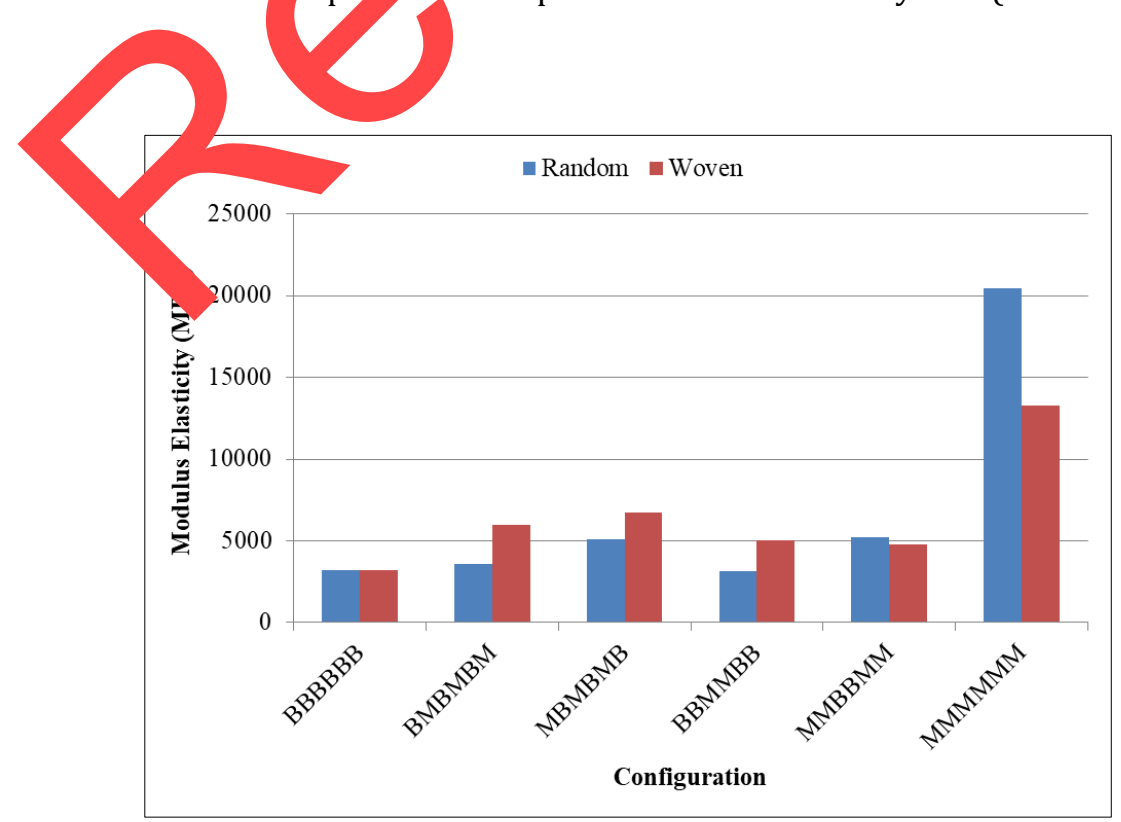

Figure 4 Elasticity modulus of polyester composite reinforced fiber hybrid. (Bamboo fiber-MASF) 
Figure 4 shows the elasticity modulus of the polyester composite reinforced fiber hybrid. It is seen that the elasticity modulus of (random and woven) arrangement, MMMMMM configuration is much higher compared to the BBBBBB configuration. The value of the composite elasticity modulus increases with the increase in the value of the maximum stress or load or the increase in the value of the tensile strength. The highest elasticity modulus by $20478 \mathrm{MPa}$ random arrangement, configuration MMMMMM and the lowest by $3224 \mathrm{MPa}$ woven arrangement, configuration BBBBBB. The modulus of elasticity for specimens with woven MASF arrangement is higher than for random. The location of MASF or bamboo fiber, by turns configurations (MBMBMB, BMBMBM) or block configuration blocks (BBMMBB, FFBBMM) affects the elasticity modulus value.

\subsection{The Microstructure of material fracture}

The results of observations with SEM of specimen fracture, with fiber random arrangement and configuration variations as shown in Figure 5.a-e. The failure (fracture) occurred in the middle area. The fracture/failure of polyester composites reinforced fiber hybrid with random arrangement MASF with the same configuration as woven arrangement MASF, shows similarities for each configuration. However, fracture of MASF does not indicate the presence of long pulled fiber as in woven MASF. It shows that the bonding of random MASF with the polyester mat $\mathrm{v}$ is better than that of woven arrangement MASF. However, because the fiber is random and short, the load transfer en tiveness is not as effective as continuous fiber as in woven arrangement MASF.

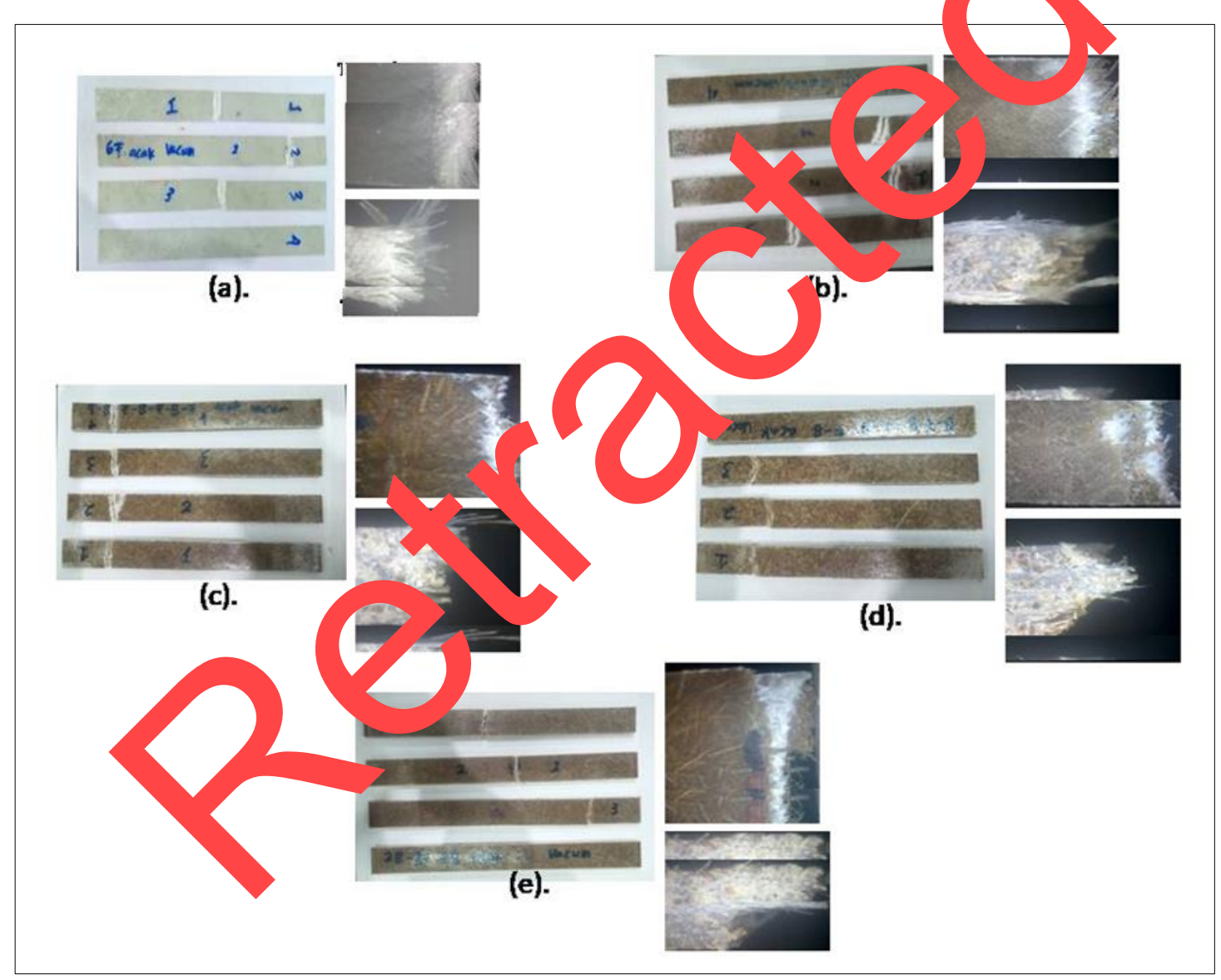

Figure 5 The Microstructure of fracture for fiber random arrangement (a). MMMMMM,

$$
\text { (b). BBBBBB, (c) MMBBMM, (d) MBMBMB, (e). BMBMBM }
$$

Figure 6.a which is a composite failure of woven fiber arrangement. Fiber failure looks stringy. Individual MASF are separated from the matrix. While Figure 6.b is a failure of bamboo fiber composites, the failure also shows the existence of bamboo fibers that are pulled out but are short and not like MASF. The larger diameter of the bamboo and its length which is not as long makes the reinforcement/load transfer less effective than MASF. In addition, the tensile strength of MASF is much higher than that of bamboo fiber, so the strength of polyester composites reinforced MASF is much higher than reinforced bamboo fiber. The microstructure of fracture for fiber configuration of each specimen is different, which may be caused by different layers or the addition of bamboo fiber or MASF. In the FFBBBFF configuration (Fig. 4.5c), 
the outer layer of MASF was seen as having longer fibers than the bamboo fiber, especially on the composite surface and in the middle, the failure of the bamboo fiber was pulled out but outer layer.

different from MASF. The outer fiberglass configuration allows for earlier (longer) reinforcement or load transfer on the surface and is very effective. So that the outer MASF arrangement is able to give better strength to the composite. In Figure 5.5d (FBFBFBF configuration), the failure of each alternating layer of fiberglass is seen as long stringy. Although it contains the same amount of fiberglass but different layers, the outermost content of fiberglass provides better strength compared to alternating arrangements. While Figure 5.5e is a photo of the failure of the woven fiberglass composite (BFBFBFB). Fiberglass failure in each layer looks longer stringy than bamboo fiber. The failure also indicated the presence of short but plucked bamboo fibers on the specimen surface and each layer but unlike MASF which was long in each alternating layer. The Microstructure of fracture for fiber configuration (BBFFFBB) as shown in Figure 5.5f. On the surface of the specimen, the failure indicated the presence of bamboo fibers experiencing early failure which were pulled out but short, the failure also indicated the presence of MASF with long fibers in the arrangement that gathered in the middle. The larger diameter of the bamboo and its length which is not as long as the MASF makes the reinforcement/load transfer less effective than fiberglass. Besides that, the strength of the composite with the outermost fiberglass content is higher than the fiberglass content that collects in the mi 'e.

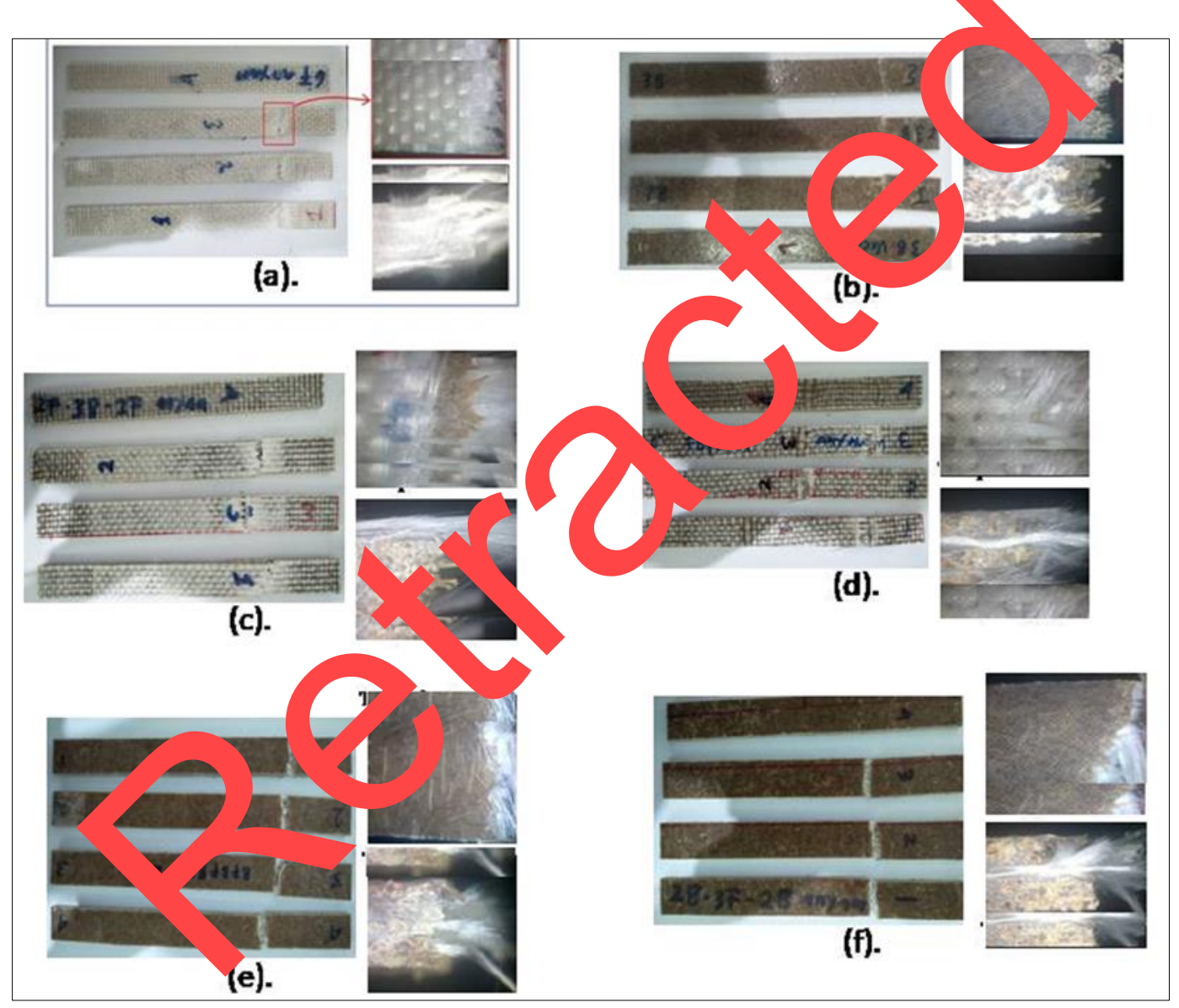

Figure 6 The Microstructure of fracture for fiber woven arrangement (a). MMMMMM,

(b). BBBBBB, (c) MMBBMM, (d) MBMBMB, (e). BMBMBM

\section{Conclusion}

Tensile strength, elasticity modulus of polyester composites reinforced MASF with woven, random arrangement is better than that of polyester composites reinforced bamboo fiber. So that in the manufacture of polyester composites reinforced hybrid fiber the use of more MASF than bamboo fiber can provide better tensile strength and elasticity modulus. The configuration with the outer MASF layer can also increase the value of tensile strength, elasticity modulus on the specimen compared to the configuration with bamboo fiber outside. The microstructure of fracture for fiber for 
each configuration of MASF content there are long fibers and also in bamboo fibers there are fibers that are pulled out. The diameter of the bamboo fiber reinforcement is larger so that the load transfer is not as effective as MASF.

\section{Compliance with ethical standards}

\section{Acknowledgments}

The intellectual and moral contributions of Prof. DR. Ir. Rudy Soenoko, Msc.Eng., Prof. DR. Eng. Ir. IGN. Wardana, Prof. Sugiman, ST., MT. among others towards the success of this work are deeply appreciated.

\section{Disclosure of conflict of interest}

No conflict of interest.

\section{References}

[1] Braga RA. Rear Bumper Laminated In Jute Fiber With Polyester Resin, Int. Journal ngineering Research and Applications www.ijera.com ISSN: 2248-9622. 2014; 4(9): 174-184.

[2] Wijoyo, Sugiyanto dan Catur Purnomo. Effect of Surface Treatment of Pineapple ber (An lus Comosus L. Merr) on Tensile Strength and Adhesion as Composite Materials, Jurnal M ca Engineering -UNS Surakarta., Indonesia. 9(2).

[3] Thiruchitrambalam M, dkk. Improving Mechanical Properties f ana Kenar Polyester Hybrid Composites Using Sodium Laulryl Sulfate Treatment, Material Physics and N. anics. o: 165-173.

[4] Annual Book of ASTM Standards, D3039, Standard Test M cun For M aical Properties Of Unreinforced And Reinforced Plastics And Electrical Insulating Material, AS 4 Stand 2 ds and Literature References for Composite Materials, 2nd ed. 1997; 34-37.

[5] Q Liu, T Stuart, M Hughes, HSS Sharma, G Lyons. A Uct al blon posites from flax-part II: the use of PEG and PVA as interfacial compatibilising agents, Coml site Pa A: Applied Science and Manufacturing. 2007; 38(5): 1403-1413.

[6] Maleque M, Yousif B, Sapuan S. I'echan prope study of pseudo-stem banana fiber reinforced epoxy composite, Arabian Journal for Scie and b ineering. 2007; 32.

[7] Makinejad Majid, Sapuan S, Ali Aidv, Ah. d D, Awdan Khalina. Thermoplastic Impact Property Improvement in Hybrid Natural Fibre Epoxy C apos Bun Beam. IOP Conference Series: Materials Science and Engineering.

[8] Do-Hyoung Kim, Hwn-Gyun Ki , HaF jung Kim. Design optimization and manufacture of hybrid glass/carbon fiber reinforced mpo te bu er be $\mathrm{n}$ for automobile vehicle, Composite Structures. 2015; 131: $742-752$.

[9] Thiruchitra alam M mnroving Mechanical Properties of Banana/Kenap Polyester Hybrid Composites Using Sodium Laulr, Sulf Trean Material Physics and Mechanics. 2009; 8: 165-173.

[10] Guangyong Sun, X cong Wang, Jianguang Fang, Tong Pang, Qing Li. Parallelized optimization design of bumper systems under mult $\mathbf{I}_{\mathbf{I}} \quad$ low-speed impact loads,Thin-Walled Structures. 2021; 167: 108197. 